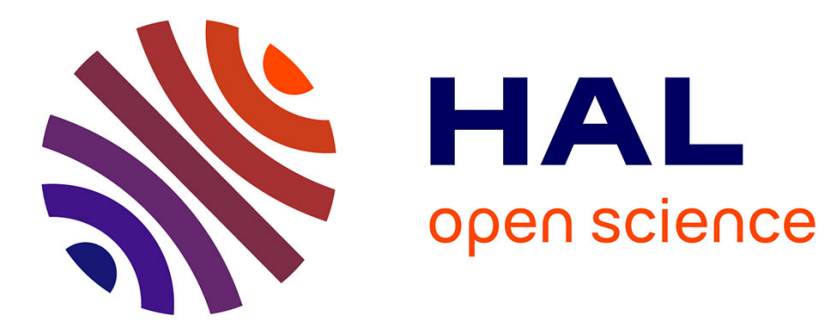

\title{
On the Grundy number of a graph
}

Frédéric Havet, Leonardo Sampaio

\section{To cite this version:}

Frédéric Havet, Leonardo Sampaio. On the Grundy number of a graph. Fifth International Symposium on Parameterized and Exact Computation (IPEC 2010), Dec 2010, Chennai, India. pp.170-179, 10.1007/978-3-642-17493-3 . inria-00532906

\section{HAL Id: inria-00532906 https://hal.inria.fr/inria-00532906}

Submitted on 5 Nov 2010

HAL is a multi-disciplinary open access archive for the deposit and dissemination of scientific research documents, whether they are published or not. The documents may come from teaching and research institutions in France or abroad, or from public or private research centers.
L'archive ouverte pluridisciplinaire HAL, est destinée au dépôt et à la diffusion de documents scientifiques de niveau recherche, publiés ou non, émanant des établissements d'enseignement et de recherche français ou étrangers, des laboratoires publics ou privés. 


\title{
On the Grundy number of a graph
}

\author{
Frédéric Havet* and Leonardo Sampaio *夫 \\ Projet Mascotte, I3S (CNRS, UNSA) and INRIA, 2004 route des lucioles, BP 93, \\ 06902 Sophia-Antipolis Cedex, France. \{fhavet,

\begin{abstract}
The Grundy number of a graph $G$, denoted by $\Gamma(G)$, is the largest $k$ such that $G$ has a greedy $k$-colouring, that is a colouring with $k$ colours obtained by applying the greedy algorithm according to some ordering of the vertices of $G$. Trivially $\Gamma(G) \leq \Delta(G)+1$. In this paper, we show that deciding if $\Gamma(G) \leq \Delta(G)$ is NP-complete. We then show that deciding if $\Gamma(G) \geq|V(G)|-k$ is fixed parameter tractable with respect to the parameter $k$.
\end{abstract}

Keywords: Colouring, Online Colouring, Greedy Colouring, NP-completeness, Fixed Parameter Complexity.

\section{Introduction}

A $k$-colouring of a graph $G=(V, E)$ is a surjective mapping $c: V \rightarrow\{1,2, \ldots, k\}$ such that $c(u) \neq c(v)$ for any edge $u v \in E$. A $k$-colouring may also be seen as a partition of the vertex set of $G$ into $k$ disjoint stable sets $S_{i}=\{v \mid c(v)=i\}$, $1 \leq i \leq k$. For convenience (and with a slight abuse of terminology), by colouring we mean either the mapping $c$ or the partition $\left(S_{1}, \ldots, S_{k}\right)$. The elements of $\{1, \ldots, k\}$ are called colours. We may sometimes refer to $S_{i}$ by its colour $i$. A graph is $k$-colourable if it admits a $k$-colouring. The chromatic number is $\chi(G)=\min \{k \mid G$ is $k$-colourable $\}$.

The most basic and most widespread on-line algorithm producing colourings is the greedy algorithm or first-fit algorithm. A greedy colouring relative to a vertex ordering $\sigma=v_{1}<v_{2}<\ldots<v_{n}$ of $V(G)$ is obtained by colouring the vertices in the order $v_{1}, \ldots, v_{n}$, assigning to $v_{i}$ the smallest positive integer not already used on its lowered-indexed neighbours. A greedy colouring has the following property:

For every $j<i$, every vertex $v$ in $S_{i}$ has a neighbour in $S_{j}$.

Otherwise $v$ would have been coloured by an integer not greater than $j$. Conversely, a colouring satisfying Property $(P)$ is a greedy colouring relative to any vertex ordering in which the vertices of $S_{i}$ precede those of $S_{j}$ when $i<j$. The Grundy number $\Gamma(G)$ is the largest $k$ such that $G$ has a greedy $k$-colouring.

\footnotetext{
* Partially supported by ANR Blanc AGAPE.

** Partially supported by CAPES/Brazil and ANR Blanc AGAPE.
} 
Hence the Grundy number and its ratio with the chromatic number measure how bad the greedy algorithm may perform on a graph.

Easily, $\chi(G) \leq \Gamma(G) \leq \Delta(G)+1$.

Determining the chromatic number of a graph is well-known to be an NP-hard problem. Similarly, determining the Grundy number of a graph is NP-hard [14]. But deciding if the chromatic number of a graph is at most $k$ is NP-complete [7] for $k \geq 3$, whereas Zaker [14] showed that for any fixed $k$, it is decidable in polynomial time if a given graph has Grundy number at most $k$. To show this, he proved that there is a finite number of graphs called $k$-atoms such that if $\Gamma(G) \geq k$ then $G$ contains a $k$-atom as an induced subgraph.

Brooks [3] proved that, for any connected graph $G, \chi(G) \leq \Delta(G)$ unless $G$ is a complete graph or an odd cycle. This implies that it can be checked in polynomial time if $\chi(G) \leq \Delta(G)$. Extensions of Brooks' Theorem have also been considered. A well-known conjecture of Borodin and Kostochka [2] states that every graph of maximal degree $\Delta \geq 9$ and chromatic number at least $\Delta$ has a $\Delta$-clique. Reed [13] proved that this is true when $\Delta$ is sufficiently large, thus settling a conjecture of Beutelspacher and Herring [1]. Further information about this problem can be found in the monograph of Jensen and Toft [10, Problem 4.8]. Generalisation of this problem has also been studied by Farzad, Molloy and Reed [6] and Molloy and Reed [11]. In particular, it is proved [11] that determining whether a graph with large constant maximum degree $\Delta$ is $(\Delta-q)$ colourable can be done in linear time if $(q+1)(q+2) \leq \Delta$. This threshold is optimal by a result of Emden-Weinert, Hougardy and Kreuter [5], since they proved that for any two constants $\Delta$ and $q \leq \Delta-3$ such that $(q+1)(q+2)>\Delta$, determining whether a graph of maximum degree $\Delta$ is $(\Delta-q)$-colourable is NP-complete.

A natural question is then to ask if an analog of Brooks' Theorem exists for the Grundy number. One may ask if it is decidable in polynomial time whether a graph $G$ satisfies $\Gamma(G)=\Delta(G)+1$. In Section 2, we answer this question in the negative by showing that this problem is NP-complete even when restricted to bipartite graphs. In particular, it implies that it is NP-hard to compute the Grundy number of a bipartite graph.

We then investigate some parameterised version of the Grundy number problem. For an introduction to parameterised algorithms and complexity, we refer the reader to [4] or [12]. Telle (See [4], Exercise 3.2.7) proved that the following problem is Fixed Parameter Tractable (FPT).

Dual of Colouring

Instance: A graph $G$ and an integer $k$.

Parameter: $k$.

Question: $\chi(G) \leq|V(G)|-k$ ?

In Section 3, we show that the following analog for greedy colouring is also FPT. 
Dual of Greedy Colouring

Instance: A graph $G$ and an integer $k$.

Parameter: $k$.

Question: $\Gamma(G) \geq|V(G)|-k$ ?

In fact, Telle showed DUAL OF COLOURING is FPT by showing that it has a quadratic kernel. Being FPT is equivalent to having a kernel but not necessarily polynomial. Then, a natural question is the following.

Problem 1. Does DuAl of Greedy Colouring have a polynomial kernel?

\section{NP-hardness results}

Before proving some complexity results, we need a preliminary lemma.

Lemma 1. Let $G$ be a graph and $x$ a vertex of $G$. If there is a greedy colouring $c$ such that $x$ is coloured $p$, then, for any $1 \leq i \leq p$, there is a greedy colouring such that $x$ is coloured $i$.

Proof. For $1 \leq i \leq p$, let $S_{i}$ be the set of vertices coloured $i$ by $c$. Then for any $1 \leq i \leq p,\left(S_{p-i+1}, \ldots, S_{p}\right)$ is a greedy $i$-colouring of $G\left[\bigcup_{j=p-i+1}^{p} S_{j}\right]$ in which $x$ is coloured $i$. This partial greedy colouring of $G$ may be extended into a greedy colouring of $G$ in which $x$ is coloured $i$.

We now show that no Brooks type theorem exists for the Grundy number.

Theorem 1. It is NP-complete to decide if a bipartite graph $G$ satisfies $\Gamma(G)=$ $\Delta(G)+1$.

Proof. The problem is trivially in NP. To show that it is also NP-complete, we present a reduction from 3-edge-colourability of 3-regular graphs, which is known to be NP-complete [8].

Let $G$ be a 3 -regular graph with $t-3$ vertices. Set $V(G)=\left\{v_{4}, v_{5}, \ldots, v_{t}\right\}$ and $E(G)=\left\{e_{1}, \ldots, e_{p}\right\}$. Let $I$ be the vertex-edge incidence graph of $G$, that is the bipartite graph with vertex set $V(I)=V(G) \cup E(G)$ in which an edge of $G$ is adjacent to its two endvertices. Also, let $M_{p, p}$ denote the graph obtained from the complete bipartite graph $K_{p, p}$ by removing a perfect matching. It can be easily checked that $\Gamma\left(M_{p, p}\right)=p$. We construct from $I$ a new bipartite graph $H$ as follows. For each vertex $e_{i} \in E(G)$, we add a copy $M_{3,3}\left(e_{i}\right)$ of $M_{3,3}$ and identify one of its vertices with $e_{i}$. We add a new vertex $r$ adjacent to all the vertices of $V(G)$. We add copies $M_{1,1}^{r}, M_{2,2}^{r}, M_{3,3}^{r}, M_{t+1, t+1}^{r}$ of $K_{1}, K_{2}, M_{3,3}$, $M_{t+1, t+1}$ and we choose arbitrary vertices $v_{1}, v_{2}, v_{3}, v_{t+1}$ respectively from each copy and add the edges $v_{1} r, v_{2} r, v_{3} r, v_{t+1} r$. Finally, for every $4 \leq i \leq t$, we do the following: for every $4 \leq j \leq i-1$, we add a copy $M_{j, j}^{i}$ of $M_{j, j}$, choose an arbitrary vertex $v_{j}^{i}$ of it and add the edge $v_{i} v_{j}^{i}$. See Figure 1 .

Observe that:

(i) $d_{H}(r)=t+1$, 


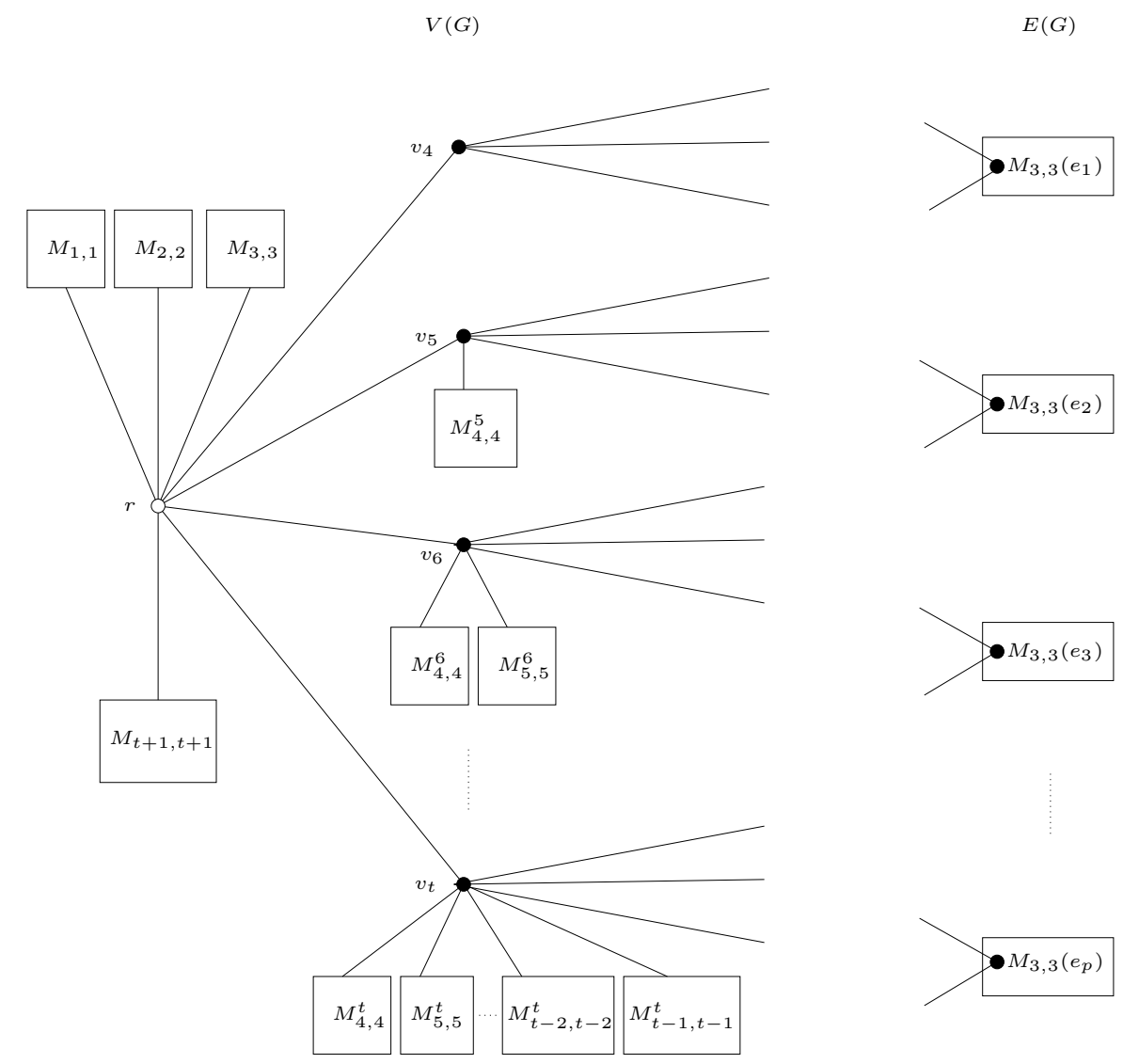

Fig. 1. The graph $H$ of the reduction in Theorem 1

(ii) $d_{H}\left(v_{i}\right)=1+(i-1)=i$, for $4 \leq i \leq t+1$.

(iii) $d_{H}\left(e_{j}\right)=4$, for $1 \leq j \leq p$, since $e_{i}$ has two neighbours in $I$ and two in $M_{3,3}\left(e_{i}\right)$.

(iv) $d_{H}\left(v_{j}^{i}\right)=j$, for $5 \leq i \leq t$ and $4 \leq j<i$, since a vertex in $M_{j, j}^{i}$ has degree $j-1$ and $v_{j}^{i}$ is adjacent to $v_{i}$.

(v) $\Delta(H)=t+1$ and the only vertices with degree $t+1$ are $r$ and $v_{t+1}$.

Let us show that $\Gamma(H)=\Delta(H)+1=t+2$ if and only if $G$ is 3-edgecolourable.

Assume first $G$ has a 3 -edge-colouring $\phi$. By Lemma 1 , for every $1 \leq i \leq p$, there is a greedy colouring of the copy of $M_{3,3}\left(e_{i}\right)$ associated with $e_{i}$ where $e_{i}$ is coloured $\phi\left(e_{i}\right)$. Then in $I$ every vertex in $V(G)$ has one neighbour of each colour in $\{1,2,3\}$. There is a greedy colouring of $M_{j, j}^{i}, 4 \leq j<i \leq t$ so that $v_{j}^{i}$ is coloured $j$. 
Then we greedily extend the union of these colourings to $v_{i}, 4 \leq i \leq t$, so that $v_{i}$ is coloured $i$. We also greedily colour $M_{1,1}^{r}, M_{2,2}^{r}, M_{3,3}^{r}, M_{t+1, t+1}^{r}$ in such a way that $r$ has one neighbour coloured $i, i \in\{1,2,3, t+1\}$. Finally, $r$ has one neighbour of each colour $j, 1 \leq j \leq t+1$. So we colour it with $t+2$.

Hence $\Gamma(H) \geq t+2$ and so $\Gamma(H)=t+2$ because $\Delta(H)=t+1$.

Let us now show that if $\Gamma(H)=t+2$ then $G$ is 3-edge-colourable. Assume that $c$ is a greedy $(t+2)$-colouring of $H$.

Claim $1\left\{c(r), c\left(v_{t+1}\right)\right\} \subseteq\{t+1, t+2\}$

Proof. Let $u$ be a vertex such that $c(u)=t+2$. Then, $u$ must have one neighbour coloured with each of the other $t+1$ colours and then $d(u) \geq t+1$. Hence, by Observation (v), $u$ is either $r$ or $v_{t+1}$.

Case 1: $u=r$.

Then, $c\left(v_{t+1}\right)=t+1$, since the only neigbours of $r$ with degree at least $t$ are $v_{t}$ and $v_{t+1}, d\left(v_{t}\right)=t$, and $v_{t}$ is adjacent to $r$ which is already coloured $t+2$.

Case 2: $u=v_{t+1}$. The only neighbour of $v_{t+1}$ that could be coloured $t+1$ is $r$, since all its neighbours in $M_{t+1, t+1}^{r}$ have degree $t$ and are adjacent to $v_{t+1}$ which is coloured $t+2$.

Claim 2 For $1 \leq i \leq t, c\left(v_{i}\right)=i$.

Proof. By Claim 1, $\left\{c(r), c\left(v_{t+1}\right)\right\} \subseteq\{t+1, t+2\}$. Since $d_{H}(r)=t+1, r$ has one neighbour coloured $i$, for each $1 \leq i \leq t$. A neighbour of $r$ which is coloured $t$ must have degree at least $t$. So, by Observation (ii), it must be $v_{t}$. And so on, by induction, we show that $c\left(v_{i}\right)=i$, for $1 \leq i \leq t$.

We now prove that $c$ induces a proper 3-edge-colouring of $G$.

Consider vertex $v_{i}, 4 \leq i \leq t$. By Claim 2 , it is coloured $i$, and by Observation(ii) it has degree equal to $i+1$. Since it is adjacent to $r$, which by Claim 1 has a colour greater than $i$, there qre only $i-1$ vertices remaining for the other $i-1$ colours. So, $v_{i}$ has exactly one neighbour coloured $j$, for each $1 \leq j \leq i-1$. Hence the three edges incident to $v_{i}$ in $G$, which are adjacent to $v_{i}$ in $I$, have different colours.

As a corollary to Theorem 1, it is NP-hard to compute the Grundy number of a bipartite graph.

Corollary 1. Given a bipartite graph $G$ and an integer $k$, it is NP-complete to decide if $\Gamma(G) \geq k$.

Theorem 1 also implies the following.

Corollary 2. Let $k \geq 0$ be a fixed integer. It is NP-complete to decide if a bipartite graph $G$ satisfies $\Gamma(G) \geq \Delta(G)+1-k$.

Proof. We present a reduction from the problem of deciding if $\Gamma(G)=\Delta(G)+1$ for a bipartite graph $G$, that we just proved to be NP-complete. Let $G$ be a graph of maximum degree $\Delta$ and $H$ the disjoint union of $G$ and the star $S_{\Delta+k+1}$ on $\Delta+k+1$ vertices $\left(S_{\Delta+k+1}\right.$ has $\Delta+k$ edges incident to a vertex). Then $\Delta(H)=$ $\Delta+k$ and $\Gamma(G)=\Gamma(H)$ because $\Gamma\left(S_{\Delta+k+1}\right)=2$. Hence $\Gamma(H) \geq \Delta(H)+1-k$ if and only if $\Gamma(G)=\Delta(G)+1$. 


\section{$3 \quad$ Fixed parameter tractability}

The aim of this section is to prove the following theorem which shows that DUAL of Greedy Colouring is FPT.

Theorem 2. Dual of Greedy Colouring can be solved in time $O\left((2 k)^{2 k} \cdot|E|+2^{2 k} k^{3 k+5 / 2}\right)$.

A vertex cover in a graph $G$ is a set $C \subseteq V(G)$ such that for every $e \in E(G)$, at least one of the endvertices of $e$ is in $C$. A vertex cover is said to be minimal if there is no vertex cover $C^{\prime} \subset C$. The complement of a graph $G$ is the graph $\bar{G}$ with the same vertex set and such that $u v \in E(\bar{G})$ if and only if $u v \notin E(G)$.

The proof of Theorem 2 may be outlined as follows. We first show that a graph $G=(V, E)$ has Grundy number at least $|V|-k$ if and only if its complement has a vertex cover with certain properties and in particular size at most $2 k$. We then give an algorithm in $O\left(k^{2 k} \cdot|E|+k^{3 k+5 / 2}\right)$ that decides if a given minimal vertex cover of $\bar{G}$ is contained in a vertex cover having such properties.

There are at most $2^{2 k}$ minimal vertex covers of size at most $2 k$ and we can enumerate them in time $O\left(2^{2 k} \cdot|V|\right)$ using a search tree (see for example Section 8.2 of [12]). Hence applying the above-mentioned algorithm for each minimal vertex cover yields an algorithm in time $O\left((2 k)^{2 k} \cdot|E|+2^{2 k} k^{3 k+5 / 2}\right)$ for DUAL of Greedy Colouring.

Lemma 2. Let $G=(V, E)$ be a graph and $k \geq 0$ an integer. Then, $\Gamma(G) \geq$ $|V|-k$ if and only if there is a vertex cover $C$ of $\bar{G}$ such that $G[C]$ admits a greedy colouring $\left(C_{1}, C_{2}, \ldots, C_{k^{\prime}}\right)$ with the following properties:

$\mathrm{P} 1:|C|-k \leq k^{\prime} \leq k$;

$\mathrm{P} 2:\left|C_{i}\right| \geq 2$, for every $1 \leq i \leq k^{\prime}$;

P3: For each $v \in V \backslash C$ and for every $1 \leq i \leq k^{\prime}$, there is $u \in C_{i}$ such that $u v \in E$.

Proof. $(\Rightarrow)$ Assume that $\Gamma(G) \geq|V|-k$ and consider a greedy $\Gamma(G)$-colouring $c$. Let $C$ be the set of vertices that are in a colour class with more than one vertex. Then $V \backslash C$ is the set of vertices that are alone in their colour classes.

Let $u$ and $v$ be two vertices in $V \backslash C$. Without loss of generality we may assume that $c(u)>c(v)$. Then, as $c$ is a greedy colouring, $u$ has a neighbour coloured $c(v)$, which must be $v$. So $u v \in E$. Hence $V \backslash C$ is clique in $G$ and so an independent set in $\bar{G}$. Consequently, $C$ is a vertex cover in $\bar{G}$.

Let $c^{\prime}$ be the greedy colouring $\left(C_{1}, C_{2}, \ldots, C_{k^{\prime}}\right)$ of $G[C]$ induced by $c$. Then, $\left|C_{i}\right| \geq 2$, for every $1 \leq i \leq k^{\prime}$, so $|C| \geq 2 k^{\prime}$. By definition of $C$, we have $\Gamma(G)=|V|-|C|+k^{\prime} \leq|V|-k^{\prime}$. Since $\Gamma(G) \geq|V|-k$, we obtain $k \geq k^{\prime} \geq|C|-k$.

Finally, let $v \in V \backslash C$ and $1 \leq i \leq k^{\prime}$. If the colour of the vertices of $C_{i}$ in $c$ is smaller than $c(v)$, then $v$ is adjacent to at least one vertex of $C_{i}$ because $c$ is greedy. If not then every vertex of $C_{i}$ is adjacent to $v$ because it is the sole vertex coloured $c(v)$. In both cases, $v$ is adjacent to at least one vertex in $C_{i}$, so $c^{\prime}$ also has Property P3. 
$(\Leftarrow)$ Let $C$ be a vertex cover of $\bar{G}$ such that there is a greedy colouring $c^{\prime}=\left(C_{1}, C_{2}, \ldots, C_{k^{\prime}}\right)$ of $G[C]$ having Properties P1, P2 and P3. One can extend $c^{\prime}$ to the entire graph $G$ by assigning $|V|-|C|$ distinct colours to the vertices of $V \backslash C$. As a consequence of $\mathrm{P} 3$ and the fact that $V \backslash C$ is an independent set in $\bar{G}$ and therefore a clique in $G$, the obtained colouring is greedy. Because of $\mathrm{P} 1$, it uses $k^{\prime}+|V|-|C| \geq(|C|-k)+|V|-|C|=|V|-k$ colours.

Let $C$ be a vertex cover of $\bar{G}$. A greedy colouring $\left(C_{1}, C_{2}, \ldots, C_{k^{\prime}}\right)$ of $G[C]$ having the Properties P1, P2 and P3 of Lemma 2 is said to be good. $C$ is suitable if $G[C]$ has a good greedy colouring. Observe that Property P1 implies that a suitable vertex cover has cardinality at most $2 k$.

Proposition 1. Let $C$ be a suitable vertex cover of $\bar{G},\left(C_{1}, C_{2}, \ldots, C_{k^{\prime}}\right)$ a good greedy colouring of $G[C]$ and $C_{m i n} \subseteq C$ a minimal vertex cover. Then for all $1 \leq i \leq k^{\prime},\left|C_{i} \backslash C_{\min }\right| \leq 1$.

Proof. Each colour class $C_{i}, 1 \leq i \leq k^{\prime}$, is an independent set of size at least 2 in $G$. So it is a clique of size at least 2 in $\bar{G}$. Since $C_{\min }$ is a vertex cover in $\bar{G}$, $\left|C_{i} \cap C_{\text {min }}\right| \geq\left|C_{i}\right|-1$, so $\left|C_{i} \backslash C_{\text {min }}\right| \leq 1$.

Lemma 3. Let $k$ be an integer, $G=(V, E)$ a graph and $C_{\text {min }}$ a minimal vertex cover of $\bar{G}$ of size at most $2 k$. It can be determined in time $O\left(k^{2 k} \cdot|E|+k^{3 k+5 / 2}\right)$ if $C_{\min }$ is contained in a suitable vertex cover $C$.

Proof. In order to determine if $C_{\min }$ is contained in a suitable vertex cover, we enumerate all possible proper colourings of $G\left[C_{\min }\right]$ with $k^{\prime}$ colours, $\left|C_{\min }\right|-k \leq$ $k^{\prime} \leq k$. For each of them, we then check in time $O\left(|E|+k^{k+5 / 2}\right)$ if it can be extended into a good greedy colouring of a suitable vertex cover. There are at most $k^{\left|C_{m i n}\right|} \leq k^{2 k}$ proper colourings of $C_{\text {min }}$ with at most $k$ colours and they can be enumerated in time $O\left(k^{2 k}\right)$. Hence the running time of our algorithm is $O\left(k^{2 k} \cdot|E|+k^{3 k+5 / 2}\right)$.

Let us now detail an algorithm that, given a proper colouring $c=\left(C_{1}, C_{2}, \ldots\right.$, $C_{k^{\prime}}$ ) of $G\left[C_{\min }\right]$, decides if it can be extended into a good greedy colouring of a suitable vertex cover in time $O\left(|E|+k^{k+5 / 2}\right)$. By Proposition 1, for such an extension at most one vertex of $V \backslash C_{\min }$ is added in each colour class.

If $c$ is a good colouring of $C_{\min }$ then we are done. So we may assume that it is not. We say that a colour class $S_{i}$ is defective with respect to a colouring $s=\left(S_{1}, \ldots, S_{l}\right)$ of $S \subseteq V$ if at least one of the following holds:

(i): $\left|S_{i}\right|<2$;

(ii): For some $j>i$, there is $v \in S_{j}$ with no neighbour in $S_{i}$;

(iii): There is $v \in V \backslash S$ such that $v$ has no neighbours in $S_{i}$.

Let $S_{i}$ be a defective colour class with respect to $s$. An $i$-candidate with respect to $s$ is a vertex $v \in V \backslash \bigcup_{j=1}^{l} S_{j}$ such that $S_{i} \cup\{v\}$ is an independent set which is not defective with respect to the colouring $\left(S_{1}, \ldots, S_{i-1}, S_{i} \cup\right.$ $\left.\{v\}, S_{i+1}, \ldots, S_{l}\right)$. We denote by $X_{s}(j)$ the set of $j$-candidates with respect to $s$ 
and $D_{s}$ the set of defective colour classes with respect to $s$. If $\left|X_{s}(j)\right| \geq k$, we say that $j$ is a colour class of type 1. Otherwise, we say that it is of type 2. It is easy to see that the set of defective colour classes of $c$ and their candidates can be computed in time $O(|E|)$.

Clearly, if $c$ can be extended into a good colouring, it means that we can place candidates to some of its colour classes and obtain a colouring without defective colour classes. Because of Proposition 1, we are only allowed to place at most one vertex in each colour class. As we will show later, the only defective colour classes that may not receive a candidate in the extension of $c$ to a good colouring are those of type 2 .

Claim 3 Let $s$ be a $k^{\prime}$-colouring of $C_{m i n}$, $i$ one of its defective colour classes and $v$ an $i$-candidate. Let $s^{\prime}$ be the extension of $s$ where we place $v$ in colour class $i$. Then, for every colour class $j \neq i, X_{s^{\prime}}(j)=X_{s}(j) \backslash\{v\}$.

Proof. First, assume that $j$ is not defective in $s$. If it does become defective in $s^{\prime}$ it is due to condition (ii), since it cannot satisfy (i) or (iii) after the insertion of $v$ in colour $i$. But then, since $j$ does not satisfy (ii) in $s, v$ is the only vertex in $i$ that may have no neighbours coloured $j$, which implies that $j$ satisfies condition (iii) in $s$, a contradiction.

Now assume that $j$ is defective in $s$. We first prove that $X_{s}(j) \backslash\{v\} \subseteq X_{s^{\prime}}(j)$. In this case, again we have that (i) and (iii) remain unchanged after the insertion of $v$, in the sense that if a vertex different from $v$ is a candidate for $j$ in $s$ because of one of these conditions, the same will happen in $s^{\prime}$. Regarding condition (iii), the only thing that may change in $s^{\prime}$ is that $v$ is now one of the vertices with no neighbours in $j$. Since $v$ is not in $C_{\text {min }}$, it is adjacent to every $j$-candidate. Then, every vertex distinct from $v$ that was a candidate for $j$ in $s$ remains a candidate for $j$ in $s^{\prime}$.

The converse, that is that every vertex in $X_{s^{\prime}}(j)$ is also in $X_{s}(j) \backslash\{v\}$, is trivial.

In particular, Claim 3 shows that the insertion of a candidate in a defective colour classes does not create a new defective colour class.

Claim 4 Let $s=\left(S_{1}, \ldots, S_{k^{\prime}}\right)$ be a $k^{\prime}$-colouring of $C_{\text {min }}$ and assume it can be extended into a good colouring $s^{\prime}=\left(S_{1}^{\prime}, \ldots, S_{k^{\prime}}^{\prime}\right)$ of a suitable vertex cover. If $j$ is a defective colour class in $s$ and $\left|S_{j}^{\prime} \backslash S_{j}\right|=0$, then $j$ is of type 2 in s. Moreover, $j$ is defective only because of (iii).

Proof. Let $j$ be such that $S_{j}^{\prime}=S_{j}$. If $j$ satisfies (i) then there is only one vertex coloured $j$ in $s^{\prime}$, and thus $s^{\prime}$ cannot be a good colouring, a contradiction. If $j$ satisfies (ii) then there is a vertex $v$ coloured $j^{\prime}>j$ with no neighbours in $S_{j}$. Since no vertices were added to $S_{j}$ in $s^{\prime}$, vertex $v$ also has no neighbours coloured $j$ in $s^{\prime}$, a contradiction. Hence $j$ can be defective only because of condition (iii). But then, if $\left|X_{s}(j)\right| \geq k$, as we add at most one vertex to each colour class when extending $s$ to $s^{\prime}$, at least one vertex in $X_{s}(j)$ is not in any colour class of $s^{\prime}$. Hence, there is a vertex that is not coloured in $s^{\prime}$ and has no neighbours with colour $j$, which implies that $j$ is a defective colour class in $s^{\prime}$, a contradiction. 
In order to determine if $c$ can be properly extended, in a first step we consider all possible extensions of the type 2 colour classes. For such a colour class of type 2 , we can choose to add to it either one of its candidates or none. By Claim 4 this later case is possible if the colour class satisfies only (iii). There are $k_{1}$ defective colour classes of type 2 , where $k_{1} \leq k$. Moreover each of these colour classes has $k_{2}$ candidates with $k_{2}<k$, and so has $k_{2}+1$ possible ways of extension: adding one of the $k_{2}$ candidates or adding none. Hence, we can enumerate all the possible extensions of the type 2 colour classes in time $O\left(k_{1}^{k_{2}+1}\right)=O\left(k^{k}\right)$. In the second step, for each possible extension, we check if the type 1 colour classes could be extended in order to obtain a good greedy colouring.

Let $c^{\prime}$ be one possible extension of $c$ as considered in the last paragraph. If a colour class $S_{i}$ of type 2 has not been extended, it may still be defective. If it is defective because it satisfies (i) or (ii), then it will remain defective after the second step in which we add some candidate to colour classes of type 1. Hence, we can stop, it will never lead to a good colouring. If it is defective because it satisfies (iii) (and only (iii)) then all the vertices $v \in V \backslash \bigcup_{j=1}^{l} S_{j}$ such that $v$ has no neighbours in $S_{i}$ must be placed into some type 1 colour class in any extension to a good colouring. In particular, they need to be candidates of at least one colour class of type 1 . We call such vertices $v$ necessary candidates.

Let $D_{1} \subseteq D_{c^{\prime}}$ be the set of defective colour classes in $c^{\prime}$ that are of type 1 in $c$. Also let $N$ be the set of necessary candidates in $c^{\prime}$ and $C$ the vertex cover given by the vertices coloured in $c^{\prime}$. Remember that a suitable vertex cover $C$ must satisfy $|C|-k \leq k^{\prime}$, and so if $|C|+\left|D_{1}\right|-k>k^{\prime}$ there is no way of properly extending $c^{\prime}$, since by Claim 4 we need to place one candidate in each of the $\left|D_{1}\right|$ defective colour classes of type 1 . The number of colour classes in $c$ is at most $k$, and after a candidate is placed in a defective colour class, the colour class is no longer defective. So, since the type 1 colour classes have at least $k$ candidates in $c$ and because of Claim 3, there are at least $\left|D_{c^{\prime}}\right|$ candidates for each of the $\left|D_{1}\right|$ defective colour classes of type 1 in $c$. As a consequence, there are enough candidates to place in each colour class in $D_{1}$. But we also have to ensure that every necessary candidate is placed in a defective colour class. This is equivalent to finding a matching in the bipartite graph $H$ with vertex set $V(H)=D_{1} \cup N$ and with edge set $E(H)=\left\{\left(i \in D_{1}, v \in N\right) \mid\right.$ vertex $v$ is an $i$-candidate $\}$ such that every vertex in $N$ is saturated. This can be done in time $O((|V(H)|+|E(H)|) \sqrt{|V(H)|})=O\left(k^{5 / 2}\right)$ by the algorithm of Hopcroft and Karp [9].

If such a matching does not exist, then we cannot properly extend $c^{\prime}$ by adding candidates to the vertices in $D_{1}$, and so we may reject $c^{\prime}$. If such a matching exists, since each type 1 colour class has more than $\left|D_{1}\right|$ candidates, we can greedily extend $c^{\prime}$ to a good colouring.

Hence one can check in time $O\left(|E|+k^{k+5 / 2}\right)$ if a proper colouring of $G\left[C_{\min }\right]$ can be extended into a good greedy colouring of $G$.

We are now able to prove Theorem 2 .

Proof (of Theorem 2). 
Let $G$ be an instance of the problem. To answer the question, we enumerate all minimal vertex covers of $\bar{G}$, and check, for each one, if it is contained in a suitable vertex cover. To enumerate all minimal vertex covers takes time $O\left(2^{2 k}\right)$. For each of these at most $2^{2 k}$ minimal vertex covers, we check if it is contained in a suitable vertex cover. By Lemma 3, it can be done in $O\left(k^{2 k} \cdot|E|+k^{3 k+5 / 2}\right)$. Hence the total running time is $O\left((2 k)^{2 k} \cdot|E|+2^{2 k} k^{3 k+5 / 2}\right)$.

\section{References}

1. A. Beutelspacher and P.-R. Hering. Minimal graphs for which the chromatic number equals the maximal degree. Ars Combin., 18:201-216, 1984.

2. O. V. Borodin and A. V. Kostochka. On an upper bound of a graph's chromatic number, depending on the graph's degree and density. J. Combinatorial Theory Ser. B, 23(2-3):247-250, 1977.

3. R. L. Brooks, On colouring the nodes of a network. Proc. Cambridge Phil. Soc. 37:194-197, 1941.

4. R. G. Downey and M. R. Fellows. Parameterized Complexity. Monographs in Computer Science. Springer-Verlag, New York, first edition, 1999.

5. T. Emden-Weinert, S. Hougardy, and B. Kreuter. Uniquely colourable graphs and the hardness of colouring graphs of large girth. Combin. Probab. Comput., 7(4):375386, 1998.

6. B. Farzad, M. Molloy, and B. Reed. $(\Delta-k)$-critical graphs. J. Combinatorial Theory Ser. B, 93(2):173-185, 2005.

7. M. R. Garey, D. S. Johnson and L. J. Stockmeyer. Some simplified NP-complete graph problems. Theoret. Comput. Sci. 1:237267, 1976.

8. I. Holyer. The NP-completeness of edge-coloring. SIAM J. Computing 2:225-231, 1981.

9. J. E. Hopcroft and R. M. Karp An $O\left(n^{5 / 2}\right)$ algorithm for maximum matchings in bipartite graphs. SIAM J. Comput. 2(4): 225-231, 1973.

10. T. R. Jensen and B. Toft. Graph coloring problems. Wiley-Interscience Series in Discrete Mathematics and Optimization. John Wiley \& Sons, Inc., New-York, 1995.

11. M. Molloy and B. Reed. Colouring graphs when the number of colours is nearly the maximum degree. In Proceedings of the Thirty-Third Annual ACM Symposium on Theory of Computing, pages 462-470 (electronic), New York, 2001. ACM.

12. R. Niedermeier. Invitation to Fixed-Parameter Algorithms, Oxford University Press, 2006.

13. B. Reed. A strengthening of Brooks' theorem. J. Combinatorial Theory Ser. B, 76(2):136-149, 1999.

14. M. Zaker. Results on the Grundy chromatic number of graphs. Discrete Math. 306(23):3166-3173, 2006. 